\title{
The halo split skin graft in the management of non-melanoma skin cancer of the leg: a retrospective study
}

\author{
Danika Fietz ${ }^{1}$, Graham Sivyer ${ }^{2}$, Denis O’Brien ${ }^{1}$, Cliff Rosendahl ${ }^{2}$
}

1 Mermaid Beach Medical Centre, Mermaid Beach, Gold Coast, Australia
2 School of Medicine, The University of Queensland, Brisbane, Australia

Key words: skin cancer, halo graft, non-melanoma skin cancer, NMSC, leg

Citation: Fietz D, Sivyer G, O’Brien D, Rosendahl C. The halo split skin graft in the management of non-melanoma skin cancer of the leg: a retrospective study. Dermatol Pract Conc. 2013;3(4):11. http://dx.doi.org/10.5826/dpc.0304a11.

Received: June 24, 2013; Accepted: September 1, 2013; Published: October 31, 2013

Copyright: $@ 2013$ Fietz et al. This is an open-access article distributed under the terms of the Creative Commons Attribution License, which permits unrestricted use, distribution, and reproduction in any medium, provided the original author and source are credited.

Funding: None.

Competing interests: The authors have no conflicts of interest to disclose.

All authors have contributed significantly to this publication.

Corresponding author: Cliff Rosendahl, PO Box 734, Capalaba, Queensland, 4157, Australia. Tel. +61 73245 3011; Fax. +61 73245 3022. Email: cliffrosendahl@bigpond.com

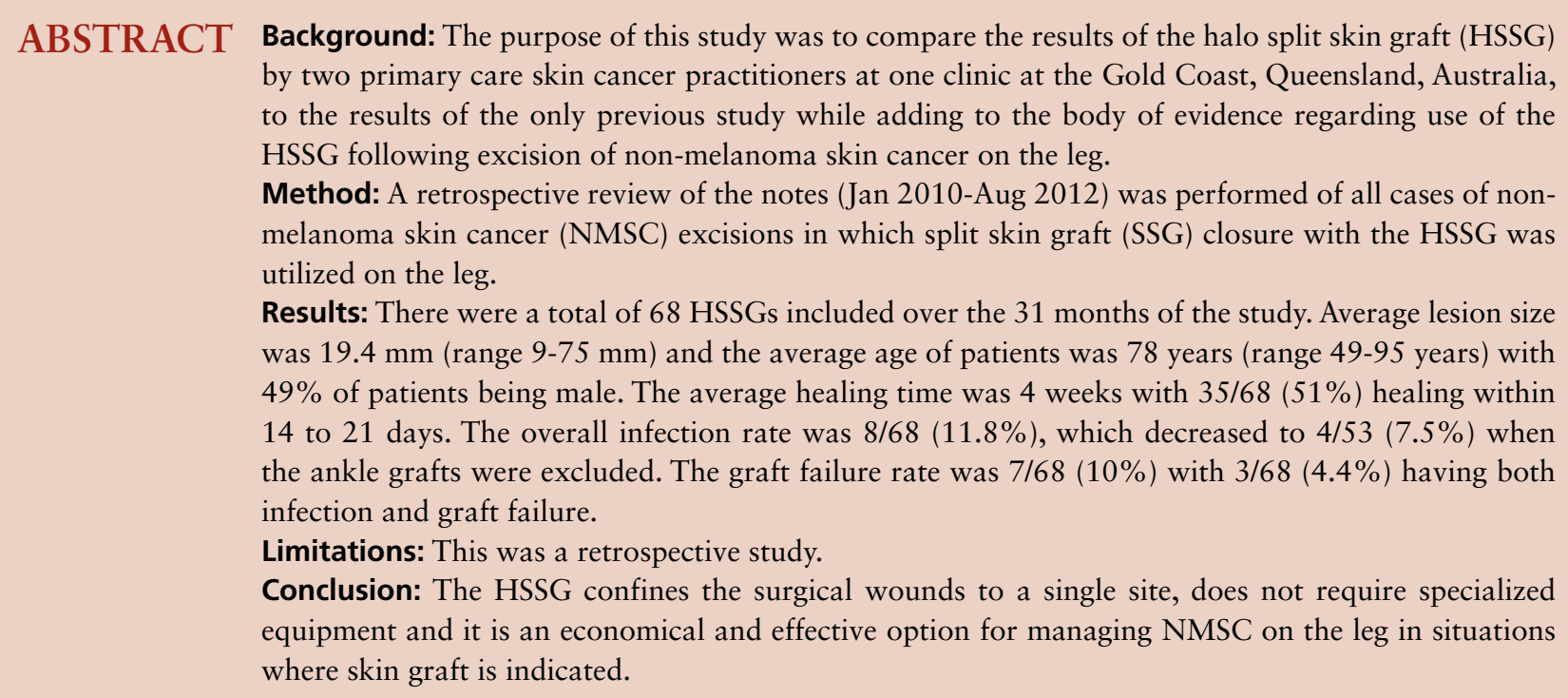

\section{Introduction}

The halo split skin graft (HSSG) was first described by Sharad Paul in 2010, with a series of 28 procedures, as a novel method for surgically managing non-melanoma skin cancer (NMSC) lesion defects on the leg (lower limb below the knee to the ankle) [1]. This technique eliminated the need for two separate surgical sites, both requiring local anesthetic and 
post-operative management. Paul also reported decreased healing times and reduced donor site pain. Because no hospitalization or specialized instruments were required, this procedure was described as cost effective. Despite the results of his study supporting these statements, a literature search has not revealed any further publications to replicate or add to the findings made by Paul in his original article. There has however been a small replication study (12 HSSG) which remains unpublished but which was presented at a Mohs surgery meeting [2]. Although that presenter described a healing rate of between 5.3-16 weeks (average 9 weeks), he remained impressed by the results and argued that the healing was much faster than wound healing via secondary intention.

Split skin grafts (SSG) have been in use since the 19th century. Conventional methods of harvesting SSG involve the use of specialized equipment such as a Weck blade (Pilling, Weck Surgical, North Carolina, USA), or power dermatome, following which the graft is sutured or stapled into place [3]. The HSSG is a type of SSG where the donor skin is harvested from the periphery of the initial circular excision.

After familiarizing themselves with the HSSG technique as published by Paul, two skin cancer primary care practitioners at one clinic commenced using the HSSG technique for NMSC surgery on the leg. They selected their patients for this procedure by choosing those lesions, which were unable to be treated by either primary or flap closure (employing either a Keystone island flap [4] or a ROM flap [5]). Previously they would have referred these patients to hospital for admission in order to have excision and SSG using the traditional methods.

The purpose of this study was to compare the results of the HSSG performed by the two practitioners at this clinic to the results of Paul [1] while contributing to the body of evidence regarding use of the HSSG after excision of NMSC on the leg.

\section{Methods}

A retrospective review of the medical records was performed of all cases of NMSC (including squamous cell carcinoma (SCC), keratoacanthoma (KA) and basal cell carcinoma (BCC) excisions by two skin cancer practitioners at a primary care clinic at the Gold Coast, Queensland, Australia, in which SSG closure with the HSSG was utilized on the leg, including the ankle. The time period chosen was from January 2010, when the practitioners began using the technique, until August 2012. The procedures included in this study were on NMSC on the leg (from below the knee to the ankle) as well as on NMSC that were sited both at the ankle and just distal to the ankle including the Achilles tendon area and also the dorsum of the foot immediately adjacent to the ankle. This group of lesions both at and just distal to the ankle were grouped together and allocated to the 'ankle' for the purpose of this study.
The demographics of the patient population treated at the clinic reflected the popularity of the region with people at retirement age. Many of these patients had multiple comorbidities including one or more of cardiovascular disease, peripheral arterial disease, diabetes mellitus, chronic renal impairment, chronic venous insufficiency and cognitive impairment. A proportion of them were consequently on multiple medications including, of significance to surgery, immunosuppressant therapy, anticoagulants and anti-platelet medications.

The HSSG cases were compiled by the researcher via a search of the Medicare billing item-numbers and then crosschecked via a manual search of the surgery booking system. The researcher entered the data collected into the excel program (EXCEL- Microsoft Corporation, Redmond, WA, USA) for further analysis.

Wound healing time was estimated using a surrogate marker being the number of weekly dressing changes. The patients were routinely seen after one week (i.e., 5-7 days post-surgery) by the registered nurse (RN) who worked exclusively in the skin cancer clinic as well as by the practitioner who had performed the surgery. The patients continued to attend the clinic approximately every seven days thereafter for wound review and dressing changes (again seen by both the $\mathrm{RN}$ and practitioner involved). This routine was altered and more frequent visits were made in the case of complications such as infection. The marker of wound healing was taken from the weekly visit that either the foam dressing was changed to a dry dressing or when the wound was left open or when a notation was made that complete wound healing had taken place. Patients that were either lost to follow-up or had their wound care carried out by nursing staff at residential facilities (where healing assessment could not be made by the investigator) were excluded from the study.

The actual size of the lesions was included for the purpose of this study and where size had not been recorded in the notes it was determined from the histopathology report. Surgical margins were marked prior to excision of the lesions according to those recommended in the clinical guidelines [6] with a minimal margin of $3 \mathrm{~mm}$ for clinically favorable BCCs and keratoacanthomas and $5 \mathrm{~mm}$ for clinically favorable SCCs. Pre-operative biopsy was routinely performed to establish the diagnosis and histological characteristics.

All cases of HSSG included had healed within the time period of the study.

\section{The HSSG Protocol}

The practitioners followed a similar protocol to Paul [1] with some minor modifications with respect to the harvesting instrument and the dressing protocol. Both practitioners chose to use a flexible shave biopsy blade (Biopsiblade-Kai Medical, Honolulu, USA) as they felt that it enabled a thin- 


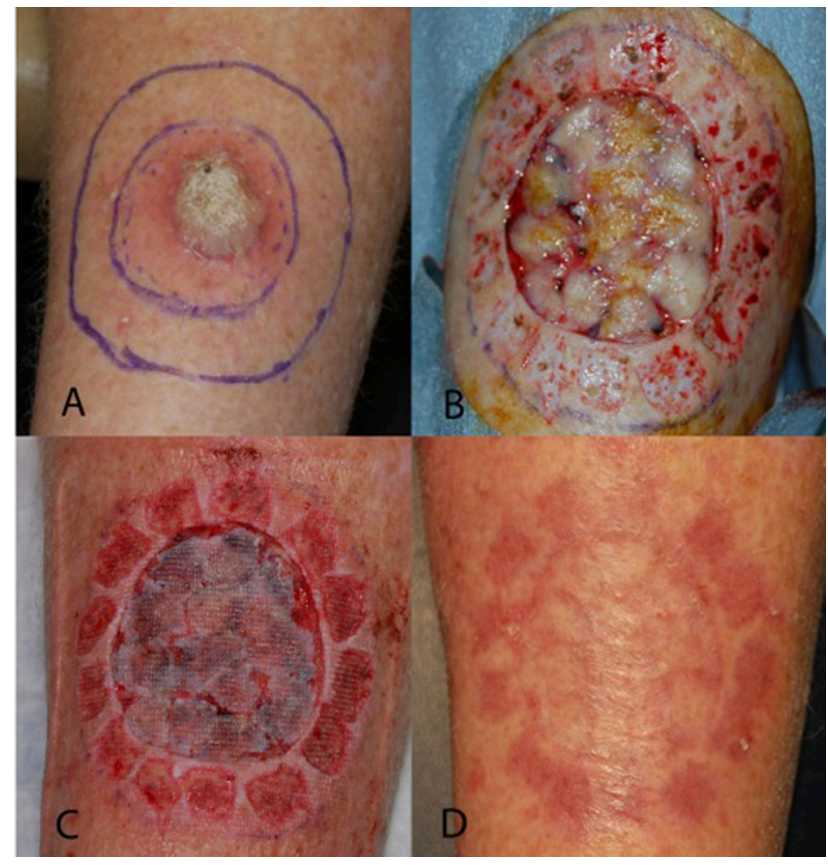

Figure 1. (A) Clinical image of a squamous cell carcinoma of the leg marked up for surgery. The inner circle indicates the planned excision margins. The annulus between the two circles is the planned donor-site. The radius of the outer circle is approximately 1.5 times the radius of the inner circle. (B) Skin has been harvested from the donor site and laid in mosaic pattern on the surgical defect. (C) Appearance of the surgical site at the time of the first dressing change seven days after surgery. (D) Appearance of the surgical site six months after surgery. [Copyright: @2013 Fietz et al.]

ner graft than that of the No. 22 scalpel blade that was used by Paul [1].

The protocol adopted required that patients wash at home in triclosan 1\% (Phisohex-Sanofi-Aventis, Paris, France) body wash for 1 week prior to the surgery. At the time of presentation for surgery, the lesion and the clinical surgical margins were marked on the skin after which the annulus, from which the graft would be harvested, was marked around the central lesion (see Figure 1A). This was measured slightly differently to the method described by Paul [1]. The radius of the actual lesion plus the surgical margin was measured then multiplied by 1.5 to reveal the new radius (i.e., radius of the lesion including the surgical margin and the annulus).

Povidone iodine 10\% (Betadine-Catalent Pharma Solutions, New Jersey, USA) skin preparation was used. If the patient was allergic to Povidone, Chlorhexidine aqueous $0.1 \%$ (Pfizer) was used instead.

The procedure was performed under local anesthetic (Lignocaine $1 \%$ with adrenaline) then after excision of the lesion including the surgical margin, a flexible shave biopsy blade was utilized to take thin shavings of skin of variable sizes (approximately $10 \times 10 \mathrm{~mm}$ each) from the annulus. These shavings were carefully placed in a mosaic fashion over the central defect until full coverage was achieved. Small bridges of intact skin were left between the donor areas of the annulus (see Figure 1B). For the majority of the study time, small bleeding areas in both the donor site and the wound bed were electrically cauterized, however a change was made later in the study period to using aluminum chloride $35 \%$ for hemostasis to the donor area only, while still using cautery to achieve hemostasis in the wound bed. No aluminum chloride was used on the wound bed at any time.

The wound was dressed with paraffin-like impregnated gauze (Cuticerin-Smith \& Nephew, London, UK), covered by a hydrocellular foam dressing (Allevyn non-adhesiveSmith \& Nephew, London, UK) and then secured firmly into place with adhesive stretchable woven tape (Fixomul-BSN Medical, Hamburg, Germany). A cotton ball was strategically placed under the tape over the center of the wound to provide support to the graft.

Tubular bandage (Tensogrip-BSN Medical, Hamburg, Germany) was then placed over the lower limb for most patients. Occasionally, for those patients with a tendency towards leg edema, a primary layer of cohesive bandage (Handy Gauze Cohesive-BSN Medical, Hamburg, Germany) was placed over the limb and dressing prior to the tubular external bandage.

Patients were given both verbal and written instructions by the RN with regards to the aftercare plan which included gentle mobilization as well as and limb elevation when sitting. Patients were instructed to keep the dressings dry until they were informed that the wound had completely healed. Patients were routinely instructed to continue taking their anticoagulants or antiplatelet medications prior to surgery. Prophylactic antibiotics were only prescribed when it was perceived that there was a high risk of infection although there was no formal protocol in this regard.

Figure 1 shows images of an SCC marked out for excision (A), immediately after harvesting and placement of the graft (B) and at one week (C) and six months (D) post-operatively.

\section{Results}

There were a total of 76 HSSG performed on the leg and ankle during the 31 months of the study. Eight were excluded due to lack of clarity in the notes or because the patients were lost to follow up leaving 68 cases of HSSG which were included in the study (SCC=45, BCC=16, KA=7). All analyzed details of all of the 68 cases are displayed in Table 1.

Overall, 36/68 (51\%) of the patients were female and the average age of patients was 78 years (range $49-95$ years). The average age for females was 79 years and for males 77 years.

The average lesion size was $19.4 \mathrm{~mm}$ (range 9-75 mm).

All lesions in this study were excised down to at least the subcutaneous tissue plane. There were $2 / 68$ (2.9\%) cases in which the histopathology report stated that a margin was positive for tumor and $3 / 68(4.4 \%)$ where margins were 
TABLE 1. Results of all data analyzed in this study

\begin{tabular}{|c|c|c|c|c|c|c|c|c|c|c|c|c|}
\hline & Age & Sex & $\begin{array}{l}\text { Size } \\
(\mathrm{mm})\end{array}$ & Site & Histology & $\begin{array}{l}\text { Dressing } \\
\text { (weeks) }\end{array}$ & $\begin{array}{c}\text { Post Op } \\
\text { Bleed }\end{array}$ & $\begin{array}{l}\text { Margins } \\
\text { Positive }\end{array}$ & $\begin{array}{c}\text { Margins } \\
\text { Inadequate }\end{array}$ & $\begin{array}{l}\text { Graft } \\
\text { Failure }\end{array}$ & Infection & $\begin{array}{c}\text { Tumour } \\
\text { Recurrence }\end{array}$ \\
\hline 1 & 83 & $\mathrm{~F}$ & 23 & Leg & SCC & 4 & & & & & & \\
\hline 2 & 76 & $\mathrm{M}$ & 20 & Leg & SCC & 2 & YES & & & & & \\
\hline 3 & 84 & $\mathrm{~F}$ & 30 & Ankle & SCC & 7 & & & & & & \\
\hline 4 & 79 & $\mathrm{M}$ & 24 & Leg & BCC & 3 & & & & & & \\
\hline 5 & 77 & $\mathrm{M}$ & 22 & Leg & BCC & 4 & & & & & & \\
\hline 6 & 81 & $\mathrm{~F}$ & 14 & Ankle & SCC & 4 & & & & & YES & \\
\hline 7 & 75 & $\mathrm{~F}$ & 32 & Ankle & SCC & 6 & & & & YES & YES & \\
\hline 8 & 80 & $\mathrm{~F}$ & 11 & Leg & SCC & 2 & & & & & & \\
\hline 9 & 76 & $\mathrm{M}$ & 25 & Ankle & BCC & 4 & YES & & & & & \\
\hline 10 & 90 & $\mathrm{~F}$ & 25 & Leg & SCC & 7 & & & & & & \\
\hline 11 & 83 & $\mathrm{~F}$ & 20 & Leg & SCC & 3 & & & & & & \\
\hline 12 & 83 & $\mathrm{~F}$ & 19 & Ankle & SCC & 2 & & & & & & \\
\hline 13 & 67 & $\mathrm{M}$ & 12 & Leg & BCC & 3 & & & & & & \\
\hline 14 & 77 & $\mathrm{~F}$ & 23 & Leg & IEC & 5 & YES & & & & & \\
\hline 15 & 74 & $\mathrm{~F}$ & 10 & Leg & BCC & 3 & & & & & & \\
\hline 16 & 58 & $\mathrm{~F}$ & 14 & Leg & SCC & 2 & & & & & & \\
\hline 17 & 77 & $\mathrm{~F}$ & 15 & Leg & KA & 3 & & & & & & \\
\hline 18 & 72 & $\mathrm{~F}$ & 17 & Leg & SCC & 3 & & & & & & YES \\
\hline 19 & 77 & $\mathrm{~F}$ & 23 & Leg & SCC & 7 & & & YES & & & \\
\hline 20 & 75 & $\mathrm{M}$ & 12 & Leg & SCC & 3 & & & & & & \\
\hline 21 & 79 & $\mathrm{~F}$ & 16 & Ankle & BCC & 3 & & & & & & \\
\hline 22 & 80 & $\mathrm{~F}$ & 14 & Leg & KA & 3 & & & & & & \\
\hline 23 & 84 & $\mathrm{~F}$ & 25 & Leg & IEC & 6 & & & & & & \\
\hline 24 & 70 & $\mathrm{~F}$ & 12 & Leg & KA & 2 & & & & & & \\
\hline 25 & 85 & $\mathrm{M}$ & 18 & Leg & SCC & 4 & & & & & & \\
\hline 26 & 92 & $\mathrm{~F}$ & 23 & Leg & SCC & 4 & & & & & & \\
\hline 27 & 87 & $\mathrm{M}$ & 14 & Leg & BCC & 3 & & & & & & \\
\hline 28 & 80 & $\mathrm{~F}$ & 25 & Leg & SCC & 3 & & & & & & \\
\hline 29 & 88 & $\mathrm{~F}$ & 16 & Ankle & BCC & 4 & & & & & & \\
\hline 30 & 79 & $\mathrm{~F}$ & 13 & Leg & SCC & 3 & & & & & & \\
\hline 31 & 81 & $\mathrm{~F}$ & 12 & Ankle & SCC & 4 & & & YES & & YES & \\
\hline 32 & 84 & $\mathrm{~F}$ & 25 & Leg & SCC & 3 & & & YES & & & \\
\hline 33 & 71 & $\mathrm{M}$ & 17 & Leg & BCC & 3 & & & & & & \\
\hline 34 & 59 & $\mathrm{M}$ & 20 & Leg & SCC & 3 & YES & & & & & \\
\hline 35 & 84 & $\mathrm{M}$ & 30 & Ankle & SCC & 3 & & & & & & \\
\hline 36 & 68 & $\mathrm{M}$ & 11 & Leg & SCC & 3 & & & & & & \\
\hline 37 & 91 & $\mathrm{M}$ & 17 & Ankle & BCC & 3 & & & & & & \\
\hline 38 & 91 & $\mathrm{M}$ & 15 & Leg & BCC & 4 & & & & & & \\
\hline 39 & 91 & $\mathrm{M}$ & 19 & Leg & IEC & 4 & & & & & & \\
\hline 40 & 83 & $\mathrm{~F}$ & 23 & Leg & SCC & 3 & & & & & & \\
\hline 41 & 81 & $\mathrm{~F}$ & 20 & Leg & SCC & 3 & & & & & & \\
\hline 42 & 74 & $\mathrm{~F}$ & 22 & Leg & SCC & 5 & & & & & & \\
\hline 43 & 79 & $\mathrm{~F}$ & 20 & Leg & KA & 3 & & & & & & \\
\hline 44 & 77 & $\mathrm{M}$ & 40 & Ankle & SCC & 3 & & & & & & \\
\hline 45 & 49 & $\mathrm{~F}$ & 20 & Leg & SCC & 4 & & & & & & \\
\hline 46 & 69 & $\mathrm{M}$ & 14 & Leg & SCC & 3 & & & & & & \\
\hline 47 & 69 & $\mathrm{M}$ & 20 & Leg & BCC & 3 & & & & & & \\
\hline 48 & 77 & M & 15 & Leg & BCC & 3 & & & & & & \\
\hline
\end{tabular}




\begin{tabular}{|c|c|c|c|c|c|c|c|c|c|c|c|c|}
\hline & Age & Sex & $\begin{array}{l}\text { Size } \\
(\mathbf{m m})\end{array}$ & Site & Histology & $\begin{array}{l}\text { Dressing } \\
\text { (weeks) }\end{array}$ & $\begin{array}{l}\text { Post Op } \\
\text { Bleed }\end{array}$ & $\begin{array}{l}\text { Margins } \\
\text { Positive }\end{array}$ & $\begin{array}{c}\text { Margins } \\
\text { Inadequate }\end{array}$ & $\begin{array}{l}\text { Graft } \\
\text { Failure }\end{array}$ & Infection & $\begin{array}{c}\text { Tumour } \\
\text { Recurrence }\end{array}$ \\
\hline 49 & 78 & $\mathrm{M}$ & 26 & Leg & SCC & 4 & & & & & & \\
\hline 50 & 65 & $\mathrm{~F}$ & 15 & Ankle & SCC & 8 & & & & YES & & \\
\hline 51 & 80 & $\mathrm{M}$ & 15 & Leg & SCC & 6 & & & & & & \\
\hline 52 & 82 & $\mathrm{~F}$ & 15 & Leg & SCC & 3 & & & & & & \\
\hline 53 & 71 & $\mathrm{~F}$ & 23 & Leg & SCC & 8 & & & & & YES & \\
\hline 54 & 76 & $\mathrm{M}$ & 19 & Leg & BCC & 3 & & & & & & \\
\hline 55 & 89 & $\mathrm{M}$ & 30 & Leg & $\mathrm{BCC}$ & 20 & & & & YES & YES & \\
\hline 56 & 75 & $\mathrm{M}$ & 10 & Leg & BCC & 2 & & & & & & \\
\hline 57 & 83 & $\mathrm{M}$ & 17 & Leg & SCC & 5 & & & & & & \\
\hline 58 & 78 & $\mathrm{M}$ & 16 & Leg & BCC & 8 & & YES & & & YES & \\
\hline 59 & 79 & $\mathrm{M}$ & 9 & Leg & SCC & 4 & & & & & & \\
\hline 60 & 95 & $\mathrm{~F}$ & 35 & Leg & SCC & 4 & & YES & & & YES & \\
\hline 61 & 95 & $\mathrm{~F}$ & 21 & Leg & SCC & 3 & & & & & & \\
\hline 62 & 68 & $\mathrm{M}$ & 11 & Leg & SCC & 4 & & & & & & \\
\hline 63 & 77 & $\mathrm{M}$ & 23 & Ankle & BCC & 6 & & & & YES & YES & \\
\hline 64 & 77 & $\mathrm{M}$ & 25 & Leg & SCC & 4 & & & & & & \\
\hline 65 & 65 & $\mathrm{M}$ & 21 & Leg & SCC & 8 & & & & YES & & \\
\hline 66 & 81 & $\mathrm{~F}$ & 15 & Leg & KA & 3 & & & & & & \\
\hline 67 & 78 & $\mathrm{M}$ & 35 & Ankle & BCC & 6 & & & & YES & & \\
\hline 68 & 78 & $\mathrm{M}$ & 15 & Ankle & SCC & 6 & & & & YES & & \\
\hline
\end{tabular}

Abbreviations used: SCC: squamous cell carcinoma; BCC: basal cell carcinoma; KA:

potentially inadequate due in each case to the deep surgical margin having tumor clearance of less than $1 \mathrm{~mm}$. Tumor recurrence occurred in $1 / 68(1.5 \%)$ and this was in spite of the fact that the histopathological report had stated that the surgical margins were clear.

The average healing time was 4 weeks. The healing times were as follows: $6 / 68(8.8 \%)$ by week $2,35 / 68(51.4 \%)$ by week 3, 51/68 (75\%) by week $4,54 / 68(79.4 \%)$ by week 5 , $60 / 68(88.2 \%)$ by week $6,63 / 68(92.6 \%)$ by week 7 and $67 / 68(98.5 \%)$ by week 8 . The last patient $(1.5 \%)$ did not heal until week 20 and was therefore considered to have had a graft failure.

The overall infection rate was $8 / 68(11.8 \%)$ and this consisted of $4 / 15(26.7 \%)$ ankle grafts and $4 / 53(7.5 \%)$ leg grafts.

The graft failure rate was $7 / 68(10 \%)$ and three of these were co-infected. Five of the infected grafts did not fail (see Table 1). Post-operative bleeding on the first day occurred in $4 / 68(5.9 \%)$, but bleeding did not result in infection or graft failure in any of these cases.

\section{Discussion}

The HSSG procedure supports the concept of early mobilisation after a SSG procedure, which is known to reduce postoperative complications such as deep vein thrombosis while showing a similar outcome in healing times when compared to the traditional post-SSG bed rest protocols $[7,8]$. In contrast to flap-closure, the HSSG does not require sutures which is arguably an advantage in the elderly where flap closure requiring any significant tension causes a risk of 'pull-through' of suture material in the often friable, fragile skin of the leg.

A SSG is generally more likely than a full thickness skin graft to survive under suboptimal conditions and can be used to cover larger defects [3] which make the HSSG procedure arguably well suited to the leg.

Compared to the study by Paul [1] this study involved a larger group of patients (68 compared to 28 ) with a greater range of ages and an older mean age (78 compared to 71 ).

While the average size of the lesion was smaller $(19.4 \mathrm{~mm}$ compared to $31 \mathrm{~mm}$ ), the size range was greater $(9-75 \mathrm{~mm}$ compared to $20-45 \mathrm{~mm}$ ). This result in this study may have been skewed by the inclusion of histopathological macro measurements (which are usually under-estimated due to specimen shrinkage in formalin) of the lesions in cases where specimen size was not stated in the pre-operative notes.

First-day post-operative bleeding occurred in 4 cases. This occurred only in graft procedures that were performed earlier in the study prior to the change to the use of Aluminum Chloride $35 \%$ as a hemostatic agent to the donor site. This would be an area where further research with respect to risks and benefits of using Aluminum Chloride as a hemostatic agent for this type of wound would be useful.

The number of lesions that did not have clear margins at the time of surgery was $2 / 68$ (2.9\%) (one BCC and one SCC). 
These cases were not routinely re-excised but were assessed on an individual basis taking into account the patient's morbidity at the time and in fact they were both managed by ongoing surveillance. In addition, margins assessed as inadequate according to current guidelines [6] occurred in another $3 / 68(4.4 \%)$ of the cases (all SCCs) and similarly these cases were managed by observation rather than re-excision.

It is acknowledged that the HSSG procedure is unique in that the skin from the surgical margin is placed over the defect in contrast to traditional SSG procedures where the skin from a remote site is used. It is also acknowledged that this is done in a non-oriented manner. For this reason it would appear to be advisable to respond to a positive margin with a HSSG procedure by total removal of the graft, in contrast to the situation where a traditional skin graft or skin flap procedure has been employed where orientation of the specimen permits targeted re-excision if a margin is positive.

Although there was no clear protocol followed for positive or inadequate margins in the practice, which is the subject of this retrospective observational study, there is a good argument for establishing a protocol for dealing with these in the future. One such protocol could include delaying SSG closure of wounds by 24-48 hours while awaiting pathology results regarding histological margins, in order to establish that clear margins have been obtained. While Mohs surgery would be optimal prior to closure, it is not affordable for most patients.

The average time taken for wound healing was longer in this study compared to that of Paul (28 days compared to 17). It is acknowledged that the deviation from the protocol of Paul may have influenced slower healing rates although this is not the explanation in the opinion of the authors. The estimation of healing time on a weekly basis using a surrogate marker as described is likely to have produced an overestimation but it was the only method available in this retrospective study.

The infection rate was higher in this study $(11.8 \%$ of all leg and ankle HSSGs compared to $0 \%$ by Paul [1]), which may have also contributed to the overall increased time taken to heal. It is known that wounds on the lower leg have a higher rate of infection than wounds on some other sites of the body, with an increased infection rate with a more distal location from the knee $[9,10]$. This is reflected in this study with the infection rate for ankle grafts being proportionately higher than that for the leg $(26.7 \%$ vs. $7.5 \%)$. It is also possible that the infection rate in this study was overestimated by the researcher due to the methods used to collect the data, by which all instances of antibiotic prescribing were classified as due to wound infection if they occurred after the time of surgery and if an alternative reason for the prescription was not clear. Other potential causes of the higher wound infection rate and slower healing times are the warmer and more humid climate of the region [10] and patient co-morbidities such as peripheral vascular disease, chronic venous insuffi- ciency and diabetes. The older population of this study may be relevant in that regard.

Graft failure was declared in any wound that took more than 8 weeks to heal or which was described in the notes as having had a graft failure. While the infection rate was $8 / 68$ and the graft failure rate similar at $7 / 68$, there were 5 infected grafts that did not fail. Ankle HSSG however were over-represented with five of the graft failures involving ankle grafts. Post-operative bleeding, which occurred in 4/68 cases, was not associated with either infection or graft failure (see Table 1). It is not known whether the slight deviations in surgical method or the difference in dressing protocol to that described by Paul [1], contributed to the graft failure rate. However it is clear that the HSSG at the ankle were not always successful.

There have been arguments expressed against using this technique for any skin cancer due to the concept of fieldcancerization, which refers to the potential for the donor skin to contain cancer cells which may cause tumor to arise at the graft site. In response to this it is a fact that flap closure also places skin from tumor margins over the surgical defect. A recurrence did occur once during this study despite the margins having been reported as clear in the histopathology report. Although this may have been an example of field cancerization it could also have been due to limitations of histopathology methodology when assessing margins using standard techniques. Specifically, Paul maintains that the HSSG is not suitable for wound closure after melanoma excision because of the risk of field cancerization [1] and HSSG was not employed in this study after melanoma excision.

The authors of this study agree with the subjective observations made by Paul comparing the HSSG compared to traditional SSG where he stated that the contour gradient was smaller, the color and texture match to surrounding skin was superior and the reported patient pain levels were less [1]. Objective measurement of these characteristics is an area of potential future research.

A major limitation of this study is that it was retrospective. It was totally reliant on the comprehensiveness of the notes that were taken by the practitioners and the nursing staff at the time of treatment and the subsequent interpretation of those notes by the researcher.

Overall, this study highlights a number of areas in which more research is desirable. A prospective randomized study of patients allocated to a traditional SSG procedure or a HSSG procedure, would be a useful future study.

\section{Conclusion}

The results of this study suggest that the HSSG is a reasonable choice for wound management after excision of NMSC on the leg when primary closure or skin-flap closure is not pos- 
sible. It allows patients to remain out of hospital, to continue to mobilize and to convalesce at home. It also confines the surgical wounds, and thus local anesthetic infiltration, surgical healing and wound dressing to a single site. The authors of this study agree with Paul that the HSSG is a technique that does not require specialized equipment and that it is an economical and effective procedure for managing NMSC on the leg when SSG is indicated.

\section{References}

1. Paul SP. 'Halo' grafting: a simple and effective technique of skin grafting. Dermatol Surg. 2010;36:115-9.

2. Parker T. (April 2011) Halo grafts: why you don't need to dread skin cancers on the lower leg anymore. Presentation at American Academy of Dermatology, 43rd annual meeting of American College of Mohs Surgery, Las Vegas, USA.

3. Robinson J, Hanke W, Siegel, D, Fratila A. Surgery of the Skin, 2nd ed. Philadelphia: Mosby Elselvier, 2010.
4. Behan FC. The keystone design perforator island flap in reconstructive surgery. ANZ J Surgery. 2003;73:112-20.

5. Dixon AJ, Dixon MP. Reducing opposed multilobed flap repair, a new technique for managing medium sized low leg defects following skin cancer surgery. Dermatol Surg. 2004;30:1406-11.

6. Basal cell carcinoma, squamous cell carcinoma (and related lesions)—a guide to clinical management in Australia. Cancer Council Australia and Australian Cancer Network, Sydney, 2008.

7. Luczak B, Ha J, Gurfinkel R. Effect of early and late mobilisation on split skin graft outcome. Australas J Dermatol. 2012;55(1): 19-21.

8. Tallon B, Oliver G. Comparison of inpatient bed rest and home convalescence following split skin grafting to the lower leg. Australas J Dermatol. 2007;48(1):11-15.

9. Dixon AJ, Dixon MP, Askew DA, Wilkinson D. Prospective study of wound infections in dermatologic surgery in the absence of prophylactic antibiotics. Dermatol Surg. 2006;32(6):819-26.

10. Heal C, Buettner P, Browning S. Risk factors for wound infection after minor surgery in general practice. Med J Aust. 2006 Sep $4 ; 185(5): 255-8$. 